Francoise Alliot Launois: None declared, René-Marc Flipo Consultant for: Honoraria from Novartis as steering committe of this survey DOI: 10.1136/annrheumdis-2019-eular.2775

\section{FRI0668 FIRST DATA OF THE TARDIS-RAREGISTRY, A NATIONWIDE BELGIAN BIOLOGIC REGISTRY}

Diederik De Cock ${ }^{1}$, Patrick Durez ${ }^{2,3}$, Dirk Elewaut ${ }^{4,5}$, Bernard Lauwerys ${ }^{2,3}$ Rene Westhovens ${ }^{1,6}$, Patrick Verschueren ${ }^{1,6}$, Royal Belgian Society for Rheumatology. ' KU Leuven, Skeletal Biology and Engineering Research Centre, Leuven, Belgium; ${ }^{2}$ Université Catholique de Louvain, Pôle de Pathologies Rhumatismales Inflammatoires et Systémiques, Institut de Recherche Expérimentale et Clinique, Brussels, Belgium; ${ }^{3}$ Cliniques Universitaires Saint-Luc, Department of Rheumatology, Brussels, Belgium; ${ }^{4}$ Ghent University, VIB Center for Inflammation Research, Ghent, Belgium; ${ }^{5}$ Ghent University Hospital, Department of Rheumatology - MRB2, Ghent, Belgium; ${ }^{6}$ University Hospitals Leuven, Department of Rheumatology, Leuven, Belgium

Background: The Tool for Administrative Reimbursement Drug Information Sharing (TARDIS) is an electronic platform combining data collection from patients with Rheumatoid Arthritis (RA) on biologic and synthetic targeted therapy, together with the submission of a request for reimbursement of this medication.

Objectives: To present the first data ever of the TARDIS-RA registry concerning the use of biologic and synthetic targeted therapy across Belgium. Methods: The Belgian TARDIS-RA registry started in April 2015. All Belgian rheumatologist were obliged after a transition period to insert patient data via the online portal when prescribing. If data of a patient are entered for the first time, previous and current use of classical synthetic (cs), targeted synthetic (ts) and biologic (b) disease modifying antirheumatic drug (DMARD) therapies is registered. Afterwards, every next bDMARD or tsDMARD initiation, prolongation and discontinuation is registered electronically in the system. Other data captured are basic demographic information such as age, gender and disease duration and disease characteristics including ESR, CRP, joint counts, disease activity scores and $\mathrm{HAQ}$.

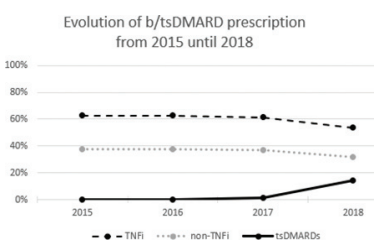

Results: At 18/01/2019, information on 80098 drug prescriptions in 13 614 patients was collected in the TARDIS-RA registry. Of these 80098 drug prescriptions, $30434(38.0 \%)$ were csDMARDs and glucocorticoids, and $49664(62.0 \%)$ were bDMARD or tsDMARD prescriptions. This last category consisted of $30380(61.2 \%)$ tumour necrosis factor inhibitor (TNFi) bDMARDs, $17 \quad 104$ (34.5\%) non-TNFi bDMARDs and $2 \quad 170$ (4.3\%) tsDMARDs. Only $642(2.3 \%)$ TNFi biosimilar prescriptions were registered. Fig1 shows the yearly evolution of bDMARD and tsDMARD prescription in Belgium from 2015 to 2018.

At first registered interaction with the rheumatologist, the 13614 patients had a median (IQR) age of 59 (50-68) years with a median (IQR) disease duration of $9(4-16)$ years; $9666(73.4 \%)$ were female. The clinical characteristics showed a median (IQR) DAS28CRP of $3.6(2.1-4.8)$ and a median (IQR) HAQ of 1.1 (0.6-1.8). Of patients at first time registration, $37.6 \%$ could be validated as being bionaïve and $62.5 \%$ as bioexperienced. Conclusion: Approximately 70000 individuals in Belgium are estimated to have a RA diagnosis. Corresponding to numbers of other western European countries, $20 \%-25 \%$ of them would be expected to receive ts/ bDMARDs. This suggests that almost the entire Belgian RA population on biologic treatment is covered by TARDIS. The registration of initiation, prolongation and discontinuation of every ts/bDMARD since 2015 combined with the simultaneous collection of demographic and clinical data, will make the TARDIS-RA registry a useful and powerful tool for the long-term drug analyses in Belgian patients with RA.

Disclosure of Interests: Diederik De Cock: None declared, Patrick Durez Speakers bureau: Bristol-Myers Squibb, Eli Lilly, Sanofi, Celltrion, Dirk Elewaut: None declared, Bernard Lauwerys: None declared, Rene Westhovens Grant/research support from: Bristol-Myers Squibb, Consultant for: Celltrion, Galapagos-Gilead, Patrick Verschueren Grant/research support from: Unrestricted Pfizer Grant for Early RA research
DOI: 10.1136/annrheumdis-2019-eular.4747

\section{FRI0669 DEPRESSION AND ANXIETY AND THEIR CLINICAL CORRELATES IN SYSTEMIC LUPUS ERYTHEMATOSUS (SLE)}

David Eldeiry ${ }^{1}$, Moe Zandy ${ }^{2}$, Oshrat Tayer-Shifman ${ }^{2}$, Sherief Marzouk ${ }^{3}$, Jiandong Su ${ }^{2}$, Zahi Touma ${ }^{2}{ }^{1}$ Royal College of Surgeons in Ireland, Medicine, Dublin, Ireland; ${ }^{2}$ University of Toronto, Rheumatology, Toronto, Canada; ${ }^{3}$ University of Toronto, Psychiatry, Toronto, Canada

Background: In our recent systematic review, we have shown a high prevalence for depression [35\% (95\% Cl: 29.9\%-40.3\%)] and anxiety [25.8\% (95\% Cl: 19.2\%-32.9\%)] in Systemic Lupus Erythematosus (SLE) ${ }^{1}$. A better understanding of the SLE phenotypic manifestations associated with depression and anxiety may lead to enhanced early diagnosis and treatment strategies.

Objectives: To determine the overall prevalence of anxiety and depression in a cohort of SLE patients, stratified by SLE-implicated organ sys tems and to study their correlates.

Methods: Patients attending the Toronto Lupus Clinic from August 2017 to January 2019 were studied. Depression and Anxiety were diagnosed with Center for Epidemiological Studies-Depression Scale (CES-D; cutoff $\geq 26$ ), BECK Depression Inventory-II (BDI-II; cut-off $\geq 18$ ), and the BECK Anxiety Inventory (BAI; cut-off $\geq 19$ ). Disease activity was measured with the SLEDAI-2k (SLE Disease Activity Index 2000). The SLE phenotypic manifestations were stratified based on the organ systems of cumulative 10-year SLEDAI-2K - skin, musculoskeletal (MSK), ocular, neuropsychiatric, and internal organ manifestations (including renal, pulmonary, immunologic, and hematologic). Separate univariate and multivariate logistic regression analyses (for depression [D], anxiety $[A]$, and anxiety and depression $[A D]$ ) were performed to study the factors associated with $A$, $D$, and $A D$, including age at enrollment, sex, ethnicity, disease duration, inception status (enrolled in the clinic within 1 year of SLE diagnosis), fibromyalgia, and SLE phenotypic manifestations, comparing their significance to the group with neither A or D.

Results: 341 patients $(89.7 \%$ female), with mean age $45.9 \pm 17.8$ years were studied. The prevalence of anxiety and depression in the cohor was $34 \%$ and $27 \%$ respectively, while $21 \%$ of the total cohort was found to have both anxiety and depression. Among the 3 outcome groups ( $A$ $D$, and $A D)$, MSK system involvement has a significantly higher prevalence when compared to the group with neither $A$ or $D$ ( $p$-values<0.05) Concurrently, skin system involvement was also significantly more prevalent among $A, D$, and $A D$ groups, in comparison to the normal group ( $p$ values $<0.05)$. Patients with anxiety had significantly higher odds of skin system involvement compared to the normal group $(\mathrm{OR}=1.81 ; 95 \% \mathrm{Cl}$ : $1.09,3.01)$. Also, patients with depression had higher odds of MSK $(\mathrm{OR}=1.94 ; 95 \% \mathrm{Cl}: 1.07,3.50)$ and skin system involvement $(\mathrm{OR}=1.79$ $95 \% \mathrm{Cl}: 1.00,3.20)$ compared to the group with neither A or D. Additionally, the odds of skin system involvement was significantly higher among patients with both $\mathrm{A}$ and $\mathrm{D}$, compared to the group with neither $(\mathrm{OR}=2.02,95 \% \mathrm{Cl}: 1.05,3.88)$. In all three models $(A, D$, and $A D)$, employment and fibromyalgia were also significant $(p$-values $<0.05)$. Age at enrolment was significant in the $D$ and $A D$ models ( $p$-value $<0.05$ ), while inception patient status [inception had a mean disease duration at study visit of $12.1 \pm 11.4$ years compared to non-inception $19.6 \pm 10.6$ years] was significant in the D model exclusively ( $p$-value $<0.05$ ).

Conclusion: SLE phenotypic manifestation, specifically those involving patients' skin or MSK systems, along with fibromyalgia, socio-demographic factors, and inception status were associated with anxiety or depression. Routine patient screening and evaluation, especially among patients with shorter disease duration, for these correlates may facilitate the diagnosis of these mental health disorders, and allow for more timely diagnosis and intervention strategies.

\section{REFERENCES:}

[1] Moustafa A, Hassanein M, Eder L, Wither JE, Fung W, Lambiris P, Touma Z. Prevalence and Metric of Depression and Anxiety in Lupus: A Systematic Review and Meta-Analyses [abstract]. Arthritis Rheumatol. 2016; 68 (suppl 10).

Disclosure of Interests: David Eldeiry: None declared, Moe Zandy: None declared, Oshrat Tayer-Shifman: None declared, Sherief Marzouk: None declared, Jiandong Su: None declared, Zahi Touma Grant/research support from: GSK Canada, Consultant for: UBC, Pfizer, Janssen, Inc DOI: 10.1136/annrheumdis-2019-eular.7595 


\section{FRI0670 HOW DO GOUT-RELATED COMORBIDITIES AND LIFESTYLE FACTORS CLUSTER IN A LARGE HEALTH SURVEY OF THE GENERAL POPULATION? - RESULTS FROM THE MALMÖ PREVENTIVE PROJECT COHORT IN SOUTHERN SWEDEN}

Tahzeeb Fatima ${ }^{1}$, Peter Nilsson ${ }^{2}$, Carl Turesson ${ }^{2}$, Mats Dehlin ${ }^{1}$, Nicola Dalbeth ${ }^{3}$ Lennart T.H. Jacobsson ${ }^{1}$, Meliha C. Kapetanovic ${ }^{2} .{ }^{1}$ University of Gothenburg, Dept of Rheumatology and Inflammation Research, Gothenburg, Sweden; ${ }^{2}$ Lund University, Dept of Clinical Sciences, Malmö, Sweden; ${ }^{3}$ University of Auckland, Dept of Medicine, Auckland, New Zealand

Background: Several factors (comorbidities and lifestyle) have been shown to be associated or predict hyperuricemia or gout. Since these factors often are closely associated with each other, they may represent a few pathophysiological pathways rather than being individually important predictors. Identifying clusters of such factors may thus lead to a better understanding of the pathways involved in increased risk of gout. Two studies have previously indicated four to five phenotype clusters in prevalent cohorts of gout patients of European ancestry ${ }^{1,2}$. However, identification of clusters of gout-associated factors in the general population is lacking.

Objectives: To identify clusters of gout-related baseline comorbidities and lifestyle factors among participants in a population-based health survey. Methods: The Malmö Preventive Project is a screening program for cardiovascular risk factors, alcohol abuse and breast cancer in Malmö, Sweden. Overall, 33,346 individuals $(67 \%$ male, mean age 45.7 years at inclusion) participated. The study population was screened between 1974 and 1992. A subset of 22,057 individuals (screening period: 1975-1992) was eligible for the cluster analysis. Agglomerative hierarchical cluster analysis was performed to group similar variables and subgroup individuals with similar characteristics, using principal component and Ward's minimum variance methods in Rv3.5.2, respectively. Variables selected to cluster were obesity $\left(B M l>30 \mathrm{~kg} / \mathrm{m}^{2}\right.$ ), renal dysfunction (eGFR $<60 \mathrm{~mL} / \mathrm{min} /$ $\left.1.73 \mathrm{~m}^{2}\right)$, diabetes mellitus $(\mathrm{DM})$, hypertension, prevalent cardiovascular disease (CVD), dyslipidemia (abnormal cholesterol or triglyceride values), pulmonary dysfunction $(\mathrm{PD}, \mathrm{FEV} / \mathrm{FVC}$ on spirometry $<0.7 \%)$, smoking and use of diuretics.

\section{A}

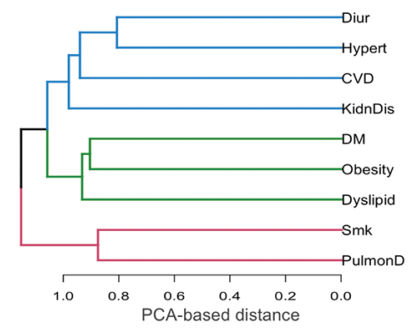

Diur: Use of Diuretics

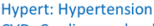

CVD: Cardiovascular disease

KidnDis: Kidney/Renal dysfunction

DM: Diabetes mellitus

Obesity: Obesity

Dyslipid: Dys/hyperlipidaemia

Smk: Smoking (current/previous)

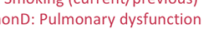

B

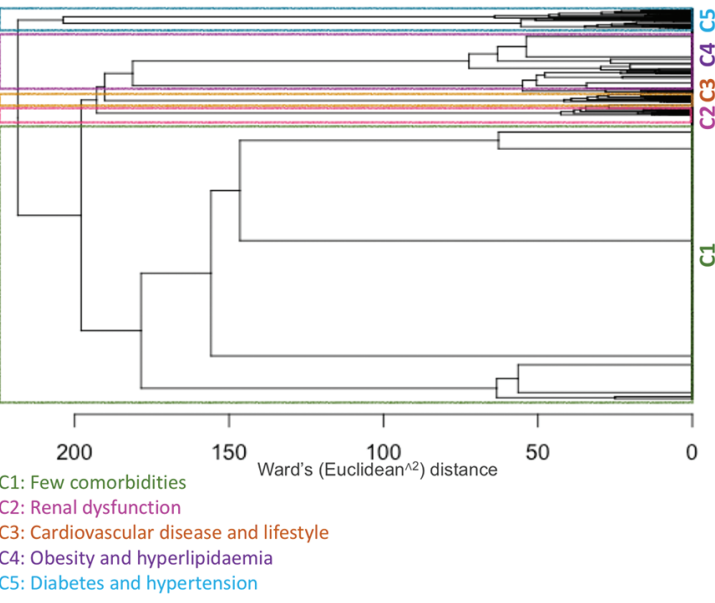

Results: Overall, $66 \%$ of the participants in the cluster analysis were males, mean age was 47 years and mean body mass index 24. Clustering of comorbidities and lifestyle factors indicated three pathways i.e. 1) mainly cardiovascular risk factors and disease, 2) variables associated with insulin resistance and 3 ) variables associated with PD (Fig; A).
Fig Results of cluster analysis illustrating (A) variable and (B) observation clustering

Five different clusters ( $\mathrm{C} 1$ to $\mathrm{C} 5$ ) were identified based on clustering of observations (Fig1; B). C1 $(n=16,063)$, mean age $=46$ years, characterized low rate of hypertension (14\%) and PD (15\%); none had obesity, kidney dysfunction, DM, CVD or dyslipidemia. C2 $(n=750$; mean age 51 years) had the highest proportions with gout (7.1\%) and kidney dysfunction $(100 \%)$, with no record of DM, CVD or use of diuretics. C3 ( $n=528$; mean age $=48$ years) had the highest rates of CVD (100\%) PD (22\%), smoking (74\%) and alcohol risk behaviour (41\%). C4 ( $n=3673$; mean age $=47$ years) had the highest percentage of males $(75 \%)$, the highest BMI (25.91) and the greatest proportions with obesity (34\%) and dyslipidemia (74\%), regular smoking (65\%) and alcohol risk behaviour (36\%). C5 $(n=1043$; mean age $=48$ years $)$ had by far the highest occurrence of DM $(51 \%)$, frequent use of diuretics (52\%), hypertension $(54 \%)$ and the highest percentage of abnormal liver enzyme levels (16\%).

Conclusion: Definition of clusters of comorbidities and lifestyle factors closely associated with gout, identified five separate "pathways" in this large health survey of the general population. "Pathways" relates to lifestyle, metabolism and specific comorbidity patterns. Further analyses will be performed to elucidate how these clusters predict diagnosed gout in this population.

\section{REFERENCES :}

[1] Richette P, et al. Ann Rheum Dis 2015; 74(1):142-47.

[2] Megan B, et al. Rheumatol 2018; 57(8):1358-63.

Disclosure of Interests: Tahzeeb Fatima: None declared, Peter Nilsson: None declared, Carl Turesson: None declared, Mats Dehlin: None declared, Nicola Dalbeth Grant/research support from: Amgen, AstraZeneca, Consultant for: Horizon, Hengrui, Kowa, Speakers bureau: Pfizer, Horizon, Janssen, AbbVie, Lennart T.H. Jacobsson Consultant for: LJ has received lecture and consulting fees from Pfizer, Abbvie, Novartis, Eli-Lily and Janssen, Meliha C Kapetanovic: None declared

DOI: 10.1136/annrheumdis-2019-eular.5642

\section{FRI0671 \\ SURVEY OF POTENTIAL TOXIC EXPOSURE IN PATIENTS WITH SYSTEMIC SCLEROSISIN RESCLE REGISTRY. A PRELIMINARY STUDY}

Mayka Freire ${ }^{1}$, Bernardo Sopeña ${ }^{1}$, Arturo González-Quintela ${ }^{1}$, Alfredo Guillén del Castillo ${ }^{2}$, Carles Tolosa ${ }^{3}$, Jose Antonio Vargas-Hitos ${ }^{4}$, Xabier Pla Salas ${ }^{5}$, Cristina González-Echávarri ${ }^{6}$, Antonio-J Chamorro ${ }^{7}$, Vicent Fonollosa $\mathrm{Pla}^{2}$, Carmen Pilar Simeón-Aznar ${ }^{2}$, On Behalf of Rescle Investigators, Autoimmune Diseases Study Group (Geas) ${ }^{8} .{ }^{1}$ Hospital Clínico Universitario de Santiago, Internal Medicine, Santiago de Compostela, A Coruña, Spain; ${ }^{2}$ Hospital Universitario Vall d'Hebron, Unit of Autoimmune Diseases, Department of Internal Medicine., Barcelona, Spain; ${ }^{3}$ Corporación Sanitaria Universitaria Parc Taulí, Department of Internal Medicine, Sabadell, Barcelona, Spain; ${ }^{4}$ Hospital Universitario Virgen de las Nieves, Department of Internal Medicine, Granada, Spain; ${ }^{5}$ Consorci Hospitalari de Vic, Unit of Systemic Autoimmune Diseases, Department of Internal Medicine., Vic, Barcelona, Spain; ${ }^{6}$ Hospital Universitario de Cruces, Autoimmune Diseases Research Unit, Department of Internal Medicine., Barajaldo, Spain; ${ }^{7}$ Complejo Asistencial Universitario de Salamanca, Internal Medicine, Salamanca, Spain; ${ }^{8}$ GEAS, Madrud, Spain

Background: Systemic sclerosis (SSc) is a systemic autoimmune disease with extremely heterogeneous clinical features and unknown etiology, although numerous studies suggest a relationship with environmental and occupational factors. So far there is little information on whether toxic substances can play a relevant role in its phenotypic expression (1)

Objectives: To analyze in a cohort of patients with SSc the proportion of patients exposed to toxic and their correlation with epidemiologic, clinical and serological data.

Methods: A survey was conducted aimed at the knowledge of the working life of patients from six centers belonging to the Spanish Scleroderma Registry (RESCLE), categorizing them in six groups: no potential exposure to toxic substances, potential exposure to silica, to hydrocarbons, to organic solvents, to mixed toxics (silica and/or hydrocarbons and/or organic solvents) and to another toxics. In all patients 87 epidemiological, clinical and analytical variables included in the registry were analyzed, carrying out a comparative study between groups.

Results: 225 SSc patients were selected. Of these, 81 patients $(36 \%)$ had worked in professions with potential risk of toxic exposure, 64 women out of the 227 included $(28 \%)$ and 17 men out of the 28 included $(60 \%)$. The toxic agent most frequently involved was silica in 29 patients $(35.8 \%)$, followed by hydrocarbons in $21(25.9 \%)$, mixture of toxic 